\title{
Turbo Receiver Design for MIMO Relay ARQ Transmissions
}

\author{
Zakaria El-Moutaouakkil( ${ }^{(1)}$, Tarik Ait-Idir ${ }^{(1,2)}$, Samir Saoudi ${ }^{(1)}$, Halim Yanikomeroglu ${ }^{(3)}$, Mounir Ghogho $^{(4)}$ \\ ${ }^{(1)}$ Institut TELECOM/TELECOM Bretagne/UMR CNRS 3192 Lab-STICC, Brest, France \\ ${ }^{(2)}$ ExceliaCom Solutions, Rabat, Morocco \\ ${ }^{(3)}$ Department of Systems and Computer Engineering, Carleton University, Ottawa, Canada \\ ${ }^{(4)}$ International University of Rabat, Sala Al Jadida, Morocco
}

Email: zakaria.elmoulieee.org

\begin{abstract}
In this paper, we investigate practical turbo receiver design for throughput-efficient relay ARQ transmissions over broadband cooperative MIMO channels. Our setup is comprised of three multiantenna nodes: a source, a destination, and a relay node operating under the amplify-and-forward half-duplex relaying mode. To attain higher system average throughput, we adopt a two-slot transmission strategy where the ARQ mechanism is activated on top of the amplifyand-forward protocol. We derive a soft sub-packet combiner allowing the destination node to jointly perform sub-packet combining (over both time-slots and multiple ARQ rounds) and frequency domain (FD) MMSE filtering. Its computational load is then smoothly relaxed via a recursive implementation alleviating the memory requirements when the number of ARQ rounds increases. Simulation results show that the proposed transmission strategy along with turbo subpacket combining at the destination side achieves significant average throughput performance gain compared with conventional ARQbased cooperative relaying, especially in situations where the relay node is close to the source node.
\end{abstract}

\section{INTRODUCTION}

Recently, the concept of cooperative relaying has emerged as an effective source of diversity and multiplexing gains, promising a wide radio coverage with a guaranteed quality of service for future wireless access systems [1], [2]. These gains are realized in a distributed fashion, in which one or several relay nodes assist the communication between a source node and a destination node. Moreover, in hybrid automatic repeat request (ARQ)-based cooperative relaying [3], relays can also act as packet retransmitters when erroneous packet decoding is detected at the destination node. This leads to a substantial source-destination link reliability enhancement compared with the classical ARQ mechanisms, as well as the potential of power saving at the source node. However, this approach may suffer from an overall throughput loss due to the half-duplex mode of operation at the relay node, because none of the above gains would be realized during the first ARQ round.

As an alternative, the ARQ mechanism can be applied on top of one of the well known amplify-and-forward (AF) or decodeand-forward (DF) relaying protocols. In which case, the destination node activates the ARQ protocol after the reception of packets from two consecutive time slots. Yet, to the best of our knowledge, no prior work has considered Nabar's protocol I [4] for realizing full rate transmission and a maximum diversity gain within each ARQ round, especially in the general case of broadband MIMO ARQ cooperative transmission.

In this paper, we address the issue of throughput-efficient relay ARQ broadband MIMO transmission, by exploiting the available relaying and ARQ diversities at the destination node. We consider that the source node follows the Relay ARQ with slot- mapping reversal (SMR) transmission strategy [5], where the ARQ mechanism is seen to be on top of the AF relaying Nabar's protocol I. In this context, we investigate practical turbo receiver design that addresses the above considerations while alleviating the computational load that may arise when the number of ARQ rounds increases. Inspired by the concept of turbo packet combining, initially introduced by Ait-Idir et al. [6] and then extended in [7] for broadband MIMO ARQ systems in the presence of cochannel interference, we propose to perform signal-level subpacket combining at the destination node jointly over both time slots and multiple ARQ rounds. Then, we introduce a recursive over the ARQ rounds implementation of this combining strategy, that considerably reduces its computational complexity. Compared with conventional ARQ-based cooperative relaying protocols, the proposed turbo receiver scheme is assessed in terms of the average throughput and its superiority is quite remarkable over the entire signal-to-noise ratio (SNR) region.

The following notation is used in this paper. Subscripts ${ }^{\mathrm{T}}$, and ${ }^{\mathrm{H}}$ denote transpose, and Hermitian transpose, respectively. $\mathbf{I}_{P}$ stands for the $P \times P$ identity matrix, while $\mathbf{0}_{P, P}$ and $\mathbf{1}_{P, P}$ correspond to the all zero and all one $P \times P$ matrices, respectively. $\mathbf{U}_{T}$ is the $T \times$ $T$ unitary matrix with entries $\left\{\mathbf{U}_{T}\right\}_{m, n}=\frac{1}{\sqrt{T}} \exp \left\{-j \frac{2 \pi m n}{T}\right\}$ for column index $n=1, \cdots, T-1$ and row index $m=1, \cdots, T-1$. $\mathbf{U}_{T, N}$ is the $T N \times T N$ matrix defined as $\mathbf{U}_{T, N}=\mathbf{U}_{T} \otimes \mathbf{I}_{N}$, where $\otimes$ stands for the Kronecker product. The notation $a \bmod b$ with $a$ and $b$ are integers means the usual modulo operation $a$ modulo- $b$. When $\mathbf{X}_{1}, \cdots, \mathbf{X}_{N} \in \mathbb{C}^{P \times Q}$, $\operatorname{diag}\left\{\mathbf{X}_{1}, \cdots, \mathbf{X}_{N}\right\} \in \mathbb{C}^{N P \times N Q}$ denotes the the block diagonal matrix holding on its diagonal matrices $\mathbf{X}_{1}, \cdots, \mathbf{X}_{N}$. The column vectors of the matrix $\mathbf{X} \in \mathbb{C}^{P \times Q}$ can be denoted by $\mathbf{x}_{1}, \cdots, \mathbf{x}_{Q}$. Therefore, we can define the vector $\underline{\mathbf{x}}$ as $\underline{\mathbf{x}}=\underset{1 \leq q \leq Q}{\operatorname{clm}}\left\{\mathbf{x}_{q}\right\}=\left[\mathbf{x}_{1}^{\mathrm{T}}, \cdots, \mathbf{x}_{Q}^{\mathrm{T}}\right]^{\mathrm{T}} \in \mathbb{C}^{P Q \times 1}$.

\section{Relay ARQ Sub-Packets Transmission Model}

\section{A. Relay $A R Q$ system with SMR strategy}

We consider a cyclic-prefix (CP)-based single-carrier (SC) block transmission between a source node $S$ and a destination node $D$ assisted by one relay node $R$. These nodes are equipped with $N_{S}, N_{D}$, and $N_{R}$ antennas, respectively, and thereby constitute the classical multi-antenna relay ARQ system depicted in Fig. 1. The link connecting each pair of nodes $\{A, B\}$ where $A \in\{S, R\}$ and $B \in\{R, D\}$ is regarded, at the $k$ th transmission, as a pointto-point frequency selective block fading MIMO channel having $L_{A B}$ (index $l=0, \cdots, L_{A B}-1$ ) independent paths. Each of these paths is characterized by its flat fading MIMO channel matrix 


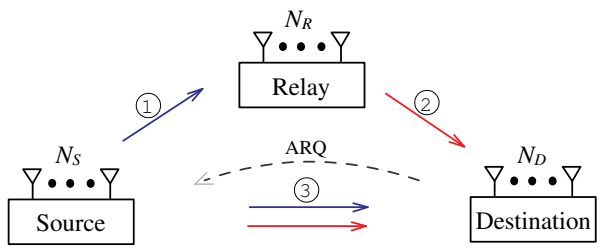

Figure 1. Multi-antenna Relay ARQ system.

$\mathbf{H}_{l}^{A B^{(k)}} \in \mathbb{C}^{N_{B} \times N_{A}}$ with independent identically distributed (i.i.d) entries drawn from zero-mean and $1 / L_{A B}$-variance circularly symmetric complex Gaussian distributions. Also, each link $\{A \rightarrow B\}$ is characterized by the channel energy $\mathrm{E}_{A B}$ capturing the effects of path loss and shadowing. To ensure high reliability and throughputefficient transmission, we assume that node $S$ uses the Relay ARQ with slots mapping reversal (SMR) re-transmission strategy proposed recently in [5]. It suggests that node $R$ performs the simple but crucial task of amplify-and-forward under the halfduplex mode. Therefore, each packet transmission $k \in\{1, \cdots, K\}$, within a maximum of $K$ ARQ rounds, is supposed to span two consecutive time slots (TS)s. Prior to the first transmission, the source node $S$ proceeds by encoding the packet of information bits $\mathbf{b}$ with the aid of a space time bit interleaved coded modulation (STBICM) encoder [8]. Then, given that $\mathcal{X}$ is the constellation set, the resultant symbol packet $^{1}$

$$
\mathbf{x} \triangleq\left[\mathbf{x}_{0}, \cdots, \mathbf{x}_{T-1}\right] \in \mathcal{X}^{N_{S} \times T},
$$

is passed through a sub-packets splitter will successively generate two $N_{S} \times \frac{T}{2}$ equally sized small packets $\mathbf{z}_{1}=\left[\mathbf{z}_{1,0}, \cdots, \mathbf{z}_{1, T / 2-1}\right]$ and $\mathbf{z}_{2}=\left[\mathbf{z}_{2,0}, \cdots, \mathbf{z}_{2, T / 2-1}\right] . T$ is the total number of channel uses in the symbol packet $\mathrm{x}$. The splitting rule is basically given by

$$
\left\{\begin{array}{ll}
\mathbf{z}_{1, t}=\mathbf{x}_{2 t} & , \quad 0 \leq t \leq \frac{T}{2}-1 \\
\mathbf{z}_{2, t}=\mathbf{x}_{2 t+1} & , \quad 0 \leq t \leq \frac{T}{2}-1
\end{array},\right.
$$

and is illustrated in general for the $k$ th transmission in Fig. 2 and Fig. 3. Accordingly, it is clear that node $S$ reverses the subpackets splitting order as a function of the ARQ round index parity. Precisely, sub-packets $\mathbf{z}_{1}$ and $\mathbf{z}_{2}$ are transmitted consecutively over the first and the second time slots, respectively, for $k$ odd, and the inverse for $k$ even. This approach, in contrast to cooperative relaying [3] where the symbol packet $\mathbf{x}$ is transmitted as such at each ARQ round, introduces a sort of correlation between the well know ARQ mechanism and the AF relaying protocol. Consequently, a substantial gain in the transmission performance is attained. It is worth noting that the considered Relay ARQ strategy follows the Chase-type ARQ mechanism. That is, when a negative acknowledgment (NACK) is sent back to $S$ at round $k$ in the case of packet decoding failure at node $D$, the source node does not intervene in changing the STBICM function, providing instead the sub-packets splitter with the same symbol packet $\mathbf{x}$ upon transmission at round $k+1$. Since no channel state information (CSI) about channel 1 (i.e., $S \rightarrow R$ link) and channel 3 (i.e., $S \rightarrow D$ link) is available at the level of node $S$, the symbol vectors $\left\{\mathbf{x}_{m} \in \mathcal{X}^{N_{S} \times 1}: m=0, \cdots, T-1\right\}$ may be chosen to have equally powered entries, hence satisfying ${ }^{2}$

${ }^{1}$ Due to the infinitely deep space-time interleaving, space and time independence between the elements of $\mathbf{x}$ is justified.

\begin{tabular}{|l|c|c|c|c|}
\hline \multirow{2}{*}{ Node Transmission } & \multicolumn{2}{|c|}{$k$ odd } & \multicolumn{2}{|c|}{$k$ even } \\
\cline { 2 - 5 } & $1^{\text {st }}$ TS & $2^{\text {nd }}$ TS & $1^{\text {st }}$ TS & $2^{\text {nd }}$ TS \\
\hline Source & $\mathbf{z}_{1}$ & $\mathbf{z}_{2}$ & $\mathbf{z}_{2}$ & $\mathbf{z}_{1}$ \\
\hline Relay & $\mathbf{y}_{R}^{(k)}$ & $\widetilde{\mathbf{y}}_{R}^{(k)}$ & $\mathbf{y}_{R}^{(k)}$ & $\widetilde{\mathbf{y}}_{R}^{(k)}$ \\
\hline \hline Destination & $\mathbf{y}_{D}^{1,(k)}$ & $\mathbf{y}_{D}^{2,(k)}$ & $\mathbf{y}_{D}^{1,(k)}$ & $\mathbf{y}_{D}^{2,(k)}$ \\
\hline
\end{tabular}

Transmission Period

Reception Period

Figure 2. SMR splitting rule at the $k$ th transmission.

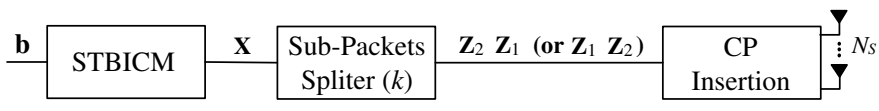

Figure 3. Source node transmitter scheme.

$$
\mathrm{E}\left[\mathbf{x}_{m} \mathbf{x}_{n}^{\mathrm{H}}\right]=\operatorname{diag}\left\{\delta_{m, n} \mathbf{I}_{1, N_{S}}\right\}, \quad \forall m, n \in\{1, \cdots, T\} .
$$

\section{B. Sub-Packets ARQ Transmission Model}

During the first TS of ARQ round $k$, node $S$ appends to $\mathbf{z}_{1}$ (here we assume that $k$ is odd, but later on we generalize our formulation despite $k$ parity) a cyclic prefix (CP) symbol word of length $L_{c p}=\max \left\{L_{S D}, L_{S R}, L_{R D}\right\}$ to avoid inter block interference (IBI), then broadcasts the resulting symbol packet simultaneously to both $R$ and $D$ nodes. After removing the CP portion at the level of node $R$, the received sequence $\mathbf{y}_{R}^{(k)}$ (or block) of signal vectors

$$
\mathbf{y}_{R, t}^{(k)}=\sqrt{\mathrm{E}_{S R}} \sum_{l=0}^{L_{S R}-1} \mathbf{H}_{l}^{S R^{(k)}} \mathbf{z}_{1,(t-l) \bmod \frac{T}{2}}+\mathbf{n}_{R, t}^{(k)}
$$

for $t=0, \cdots, T / 2-1$, is amplified to have unit average energy per each entry. Therefore, the resulting sequence $\widetilde{\mathbf{y}}_{R}^{(k)}$ is made of signal vectors $\tilde{\mathbf{y}}_{R, t}^{(k)}=\gamma \mathbf{y}_{R, t}^{(k)}$, where $\gamma=1 / \sqrt{N_{S} \mathrm{E}_{S R}+N_{0}}$. As for node $R$, the destination node $D$ performs CP removal at first, then passes the received sequence $\mathbf{y}_{D}^{1,(k)}$ of signal vectors ${ }^{3}$

$$
\mathbf{y}_{D, t}^{1,(k)}=\sqrt{\mathrm{E}_{S D}} \sum_{l=0}^{L_{S D}-1} \mathbf{H}_{1, l}^{S D^{(k)}} \mathbf{z}_{1,(t-l) \bmod \frac{T}{2}}+\mathbf{n}_{D, t}^{1,(k)},
$$

for $t=0, \cdots, T / 2-1$, to the receiver although it remains unprocessed until the end of the second slot. Note that the exponent $i \in\{1,2\}$ in $\mathbf{y}_{D}^{i,(k)}$ denotes the TS index since the $\{S \rightarrow D\}$ link is involved in transmission over two consecutive time slots. For the second TS, node $R$ forwards $\widetilde{\mathbf{y}}_{R}^{(k)}$ to the destination node $D$ while node $S$ is transmitting $\mathbf{z}_{2}$. Hence, the destination node will see during the second slot a superposition of two transmitted blocks $\widetilde{\mathbf{y}}_{R}^{(k)}$ and $\mathbf{z}_{2}$ after deleting the CP portion of length $L_{c p}$ being added to both of them. The received sequence $\mathbf{y}_{D}^{2,(k)}$ made of signal vectors $\left\{\mathbf{y}_{D, t}^{2,(k)}: t=0, \cdots, T / 2-1\right\}$ at the level of node $D$ can therefore be expressed as

$$
\begin{aligned}
\mathbf{y}_{D, t}^{2,(k)=} & \gamma \sqrt{\mathrm{E}_{S R} \mathrm{E}_{R D}} \sum_{l=0}^{L_{S R D}-1} \mathbf{H}_{l}^{S R D^{(k)}} \mathbf{z}_{1,(t-l) \bmod \frac{T}{2}}+ \\
& \sqrt{\mathrm{E}_{S D}} \sum_{l=0}^{L_{S D}-1} \mathbf{H}_{2, l}^{S D^{(k)}} \mathbf{z}_{2,(t-l) \bmod \frac{T}{2}}+\mathbf{n}_{D, t}^{\mathrm{res},(k)},
\end{aligned}
$$

\footnotetext{
${ }^{2} \delta_{m, n}$ is the Kronecker symbol that equals either to 1 if $m=n$ or 0 otherwise. ${ }^{3}$ For the sake of simplicity, we assume that the noise at the respective receiving node $B \in\{R, D\}$ follows a Gaussian distribution $\mathcal{N}\left(\mathbf{0}_{N_{B} \times 1}, N_{0} \mathbf{I}_{N_{B}}\right)$.
} 
where the channel matrix $\mathbf{H}_{l}^{S R D^{(k)}}$ is computed, for $l=$ $0, \cdots, L_{S R D}-1$ with $L_{S R D}=L_{S R}+L_{R D}-1$, as the twodimensional convolution of channel matrices $\mathbf{H}_{l}^{R D^{(k)}}$ and $\mathbf{H}_{l}^{S R^{(k)}}$.

$$
\mathbf{n}_{D, t}^{\mathrm{res},(k)}=\gamma \sqrt{\mathrm{E}_{R D}} \sum_{l=0}^{L_{R D}-1} \mathbf{H}_{l}^{R D^{(k)}} \mathbf{n}_{R,(t-l) \bmod \frac{T}{2}}^{(k)}+\mathbf{n}_{D, t}^{2,(k)}
$$

is the residual additive noise in $\mathbf{y}_{D, t}^{2,(k)}$ whose conditional covariance matrix, given that node $D$ has perfect knowledge of channel 2 (i.e., $R \rightarrow D$ link), is expressed as

$$
\begin{aligned}
\Theta_{\mathbf{n}_{D, t}^{\mathrm{res},(k)}} \mid\left\{\mathbf{H}_{l}^{R D^{(k)}}\right\} & = \\
N_{0} & {\left[\mathbf{I}_{N_{D}}+\frac{\mathrm{E}_{R D}}{N_{S} \mathrm{E}_{S R}+N_{0}} \sum_{l=0}^{L_{R D}-1} \mathbf{H}_{l}^{R D^{(k)}} \mathbf{H}_{l}^{R D^{(k)}}\right] . }
\end{aligned}
$$

To ensure spatial and temporal noise whitening at the destination node $D$ during the second TS, we rather consider the outputs $\tilde{\mathbf{y}}_{D, t}^{2,(k)}=\mathbf{F} \mathbf{y}_{D, t}^{2,(k)}$ of the whitening filter $\mathbf{F}=\mathbf{L}^{-1}$, where $\mathbf{L}$ results from the following Cholesky factorization

$$
\Theta_{\widetilde{\mathbf{n}}_{D, t}^{2,(k)}} \mid\left\{\mathbf{H}_{l}^{R D^{(k)}}\right\}=N_{0} \mathbf{L} \mathbf{L}^{\mathrm{H}} .
$$

The resulting sequence $\widetilde{\mathbf{y}}_{D}^{2,(k)}=\left\{\widetilde{\mathbf{y}}_{D, t}^{2,(k)}: t=0, \cdots, T / 2-1\right\}$ is then passed to the receiver, which in turn builds up the augmented size signal vector

$$
\mathbf{y}_{D, t}^{\mathrm{equ}^{(k)}}=\left[\begin{array}{c}
\mathbf{y}_{D, t}^{1,(k)} \\
\widetilde{\mathbf{y}}_{D, t}^{2,(k)}
\end{array}\right] \in \mathbb{C}^{2 N_{D} \times 1},
$$

for $t=0, \cdots, T / 2-1$, corresponding to reception over two consecutive time slots. Equation (7) can be re-written explicitly as

$$
\mathbf{y}_{D, t}^{\mathrm{equ}^{(k)}}=\sum_{l=0}^{L_{e q u}-1} \mathbf{H}_{l}^{\mathrm{equ}^{(k)}} \mathbf{z}_{(t-l) \bmod \frac{T}{2}}+\mathbf{n}_{D, t}^{\mathrm{equ}^{(k)}}
$$

where the equivalent channel matrix $\mathbf{H}_{l}^{\text {equ }^{(k)}} \in \mathbb{C}^{2 N_{D} \times 2 N_{S}}$ has been carefully introduced as a $k$-parity dependent matrix having the following form

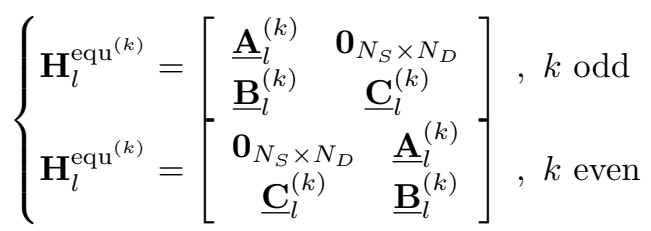

In fact, the splitting rule described earlier now becomes transparent to node $D$ as no matter node $S$ has to send firstly sub-packet $\mathbf{z}_{1}$ or $\mathbf{z}_{2}$, the issue is translated into perceiving differently the equivalent channel matrix $\mathbf{H}_{l}^{\mathrm{equ}^{(k)}}$ at each ARQ round $k$. The intermediate channel matrices $\underline{\mathbf{A}}_{l}^{(k)}, \underline{\mathbf{B}}_{l}^{(k)}$, and $\underline{\mathbf{C}}_{l}^{(k)}$ are defined as

$$
\begin{cases}\underline{\mathbf{A}}_{l}^{(k)}=\sqrt{\mathrm{E}_{S D}} \mathbf{H}_{1, l}^{S D^{(k)}} & ; 0 \leq l \leq L_{S D}-1 \\ \underline{\mathbf{A}}_{l}^{(k)}=\mathbf{0}_{N_{D} \times N_{S}} & ; l \leq L_{S D} \\ \underline{\mathbf{B}}_{l}^{(k)}=\gamma \sqrt{\mathrm{E}_{S R} \mathrm{E}_{R D}} \mathbf{F} \mathbf{H}_{l}^{S R D^{(k)}} & ; 0 \leq l \leq L_{S R D}-1 \\ \underline{\mathbf{B}}_{l}^{(k)}=\mathbf{0}_{N_{D} \times N_{S}} & ; l \leq L_{S R D} \\ \underline{\mathbf{C}}_{l}^{(k)}=\sqrt{\mathrm{E}_{S D}} \mathbf{F} \mathbf{H}_{2, l}^{S D^{(k)}} & ; 0 \leq l \leq L_{S D}-1 \\ \underline{\mathbf{C}}_{l}^{(k)}=\mathbf{0}_{N_{D} \times N_{S}} & ; l \leq L_{S D}\end{cases}
$$

From (8), it is quit straitforward to infer that the two-slots transmission strategy used by node $S$ allows us to come up with a virtual point-to-point $2 N_{D} \times 2 N_{S}$ MIMO system. Both ends of this system form a quasi-static frequency selective fading MIMO channel having $L_{e q u}=\max \left\{L_{S R D}, L_{S D}\right\}$ independent paths, and time-invariant over a duration of $\frac{T}{2}$ channel uses. The virtual channel input symbol vector at channel use $t$ is expressed as

$$
\mathbf{z}_{t} \triangleq\left[\begin{array}{c}
\mathbf{z}_{1, t} \\
\mathbf{z}_{2, t}
\end{array}\right] \triangleq\left[\begin{array}{c}
\mathbf{z}_{t}^{(1)} \\
\vdots \\
\mathbf{z}_{t}^{\left(2 N_{S}\right)}
\end{array}\right] \in \mathcal{X}^{2 N_{S} \times 1},
$$

while the additive white Gaussian noise vector $\mathbf{n}_{D, t}^{\mathrm{equ}^{(k)}}$ is explicitly given by

$$
\mathbf{n}_{D, t}^{\left.\mathrm{equ}^{(k)}\right)} \triangleq\left[\begin{array}{c}
\mathbf{n}_{D, t}^{1,(k)} \\
\mathbf{F n}_{D, t}^{\text {res, }(k)}
\end{array}\right] \sim \mathcal{N}\left(\mathbf{0}_{2 N_{D} \times 2 N_{D}}, N_{0} \mathbf{I}_{2 N_{D}}\right) .
$$

In the sequel, we will refer to $\mathbf{z}_{t}^{(i)} \in \mathcal{X}$ as being virtually the transmitted symbol by node $S$ at channel use $t=0, \cdots, T / 2-1$ from antenna $i=1, \cdots, 2 N_{S}$, and no longer look at (1). Once signal vector $\mathbf{y}_{D, t}^{\text {equ }}{ }^{(k)}$ is constructed, it is then used jointly with all the previously received signals $\mathbf{y}_{D, t}^{\mathrm{equ}^{(k-1)}}, \cdots, \mathbf{y}_{D, t}^{\mathrm{equ}^{(1)}}$ kept in memory, to form the $2 N_{D} k \times 1$ equivalent received signal vector ${ }^{4}$

$$
\underbrace{\left[\begin{array}{c}
\mathbf{y}_{D, t}^{\mathrm{equ}^{(1)}} \\
\vdots \\
\mathbf{y}_{D, t}^{\mathrm{equ}^{(k)}}
\end{array}\right]}_{\overline{\mathbf{y}}_{D, t}^{\text {equ }, k}}=\sum_{l=0}^{L_{e q u}-1} \underbrace{\left[\begin{array}{c}
\mathbf{H}_{l}^{\mathrm{equ}^{(1)}} \\
\vdots \\
\mathbf{H}_{l}^{\mathrm{equ}^{(k)}}
\end{array}\right]}_{\overline{\mathbf{H}}_{l}^{\text {equ }, k}} \mathbf{z}_{(t-l) \bmod \frac{T}{2}}+\underbrace{\left[\begin{array}{c}
\mathbf{n}_{D, t}^{\mathrm{equ}^{(1)}} \\
\vdots \\
\mathbf{n}_{D, t}^{\mathrm{equ}^{(k)}}
\end{array}\right]}_{\overline{\mathbf{n}}_{D, t}^{\text {equ }, k}},
$$

for $t=0, \cdots, T / 2-1$, which will serve to decode the transmitted packet of information bits $\mathbf{b}$. If the decoding outcome is erroneous and $k<K$, node $D$ send a NACK feedback to $S$ in order to start another ARQ round $k+1$. However, a positive acknowledgment (ACK) may be sent to $S$ by node $D$ if successful decoding is detected, in which case node $S$ should move on to the next symbol packet. We say that our system presents an error when the ARQ round $K$ is reached, i.e. $k=K$, and the decoding outcome is still erroneous.

\section{Turbo PACKet Combining Receiver Design}

In this section, inspired by [7], we derive a low complexity recursive over the ARQ rounds turbo receiver. Our main scope is on the design of a soft sub-packet combiner allowing for intersymbol interference and multiple-access interference joint cancellation from the virtual $2 N_{D} k \times 2 N_{S}$ MIMO ARQ channel (12), and signal-level sub-packet combining (referring to the combining strategy illustrated in (7) and (12)). Both tasks are jointly realized in the frequency domain using the MMSE filtering criterion. The building blocks that constitute the proposed receiver scheme are shown in Fig. 4.

\footnotetext{
${ }^{4}$ An obvious practical issue related to constructing signal vector $\overline{\mathbf{y}}_{D, t}^{e q u, k}$ and channel matrix $\overline{\mathbf{H}}_{l}^{e q u, k}$ at round $k$, is that they are built upon those received and kept in memory at round $k-1$. Therefore, the demand in terms of storage memory size gradually increases with the ARQ delay, i.e., number of ARQ rounds. This issue is addressed in Section III-C, and smoothly relaxed to keeping in memory only fixed and small size signal vectors and channel matrices at each ARQ round.
} 


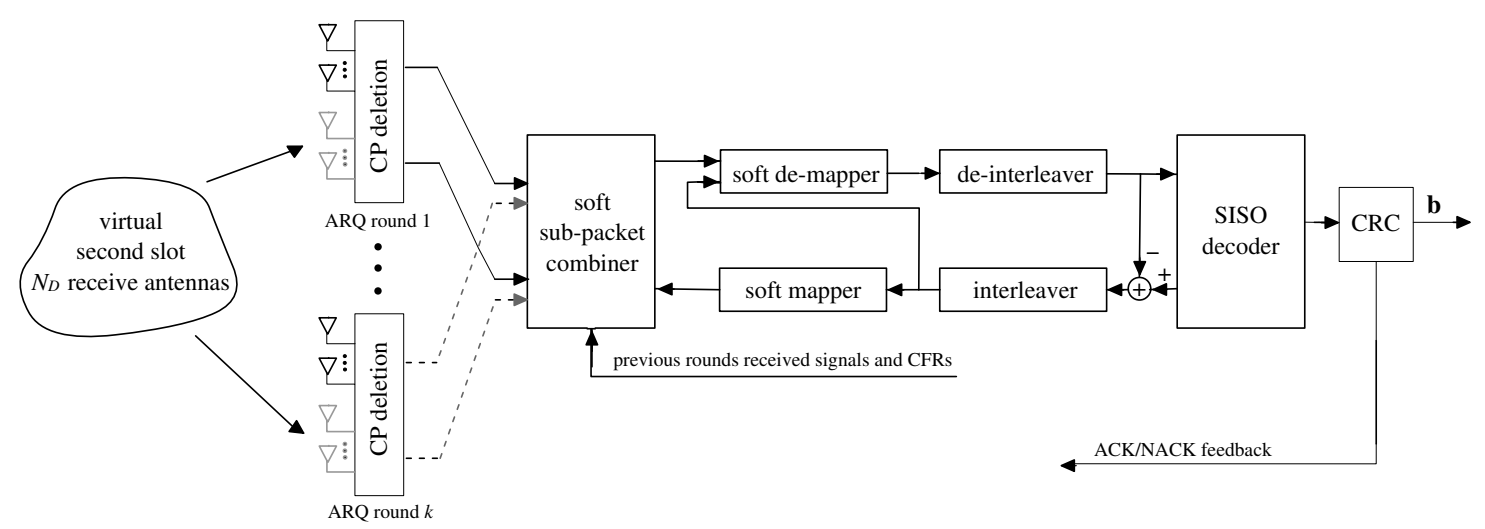

Figure 4. Building blocks of the proposed turbo receiver.

\section{A. Brief Description of the Concept}

The processing of each received sequence of signal vectors $\overline{\mathbf{y}}_{D}^{\text {equ }, k}=\left\{\overline{\mathbf{y}}_{D, t}^{\text {equ }, k}: t=0, \cdots, T / 2-1\right\}$, resulting from the joint sub-packet combination over both time slots and multiple ARQ rounds, relies on exchanging (in an iterative fashion) extrinsic information about coded and interleaved bits between the soft subpacket combiner and the SISO decoder. First of all, assuming that node $D$ has perfect knowledge of round $1, \cdots, k-1$ equivalent CSIs, the soft sub-packet combiner produces a soft estimate $\underline{\widetilde{z}}^{(k)}$ about $\underline{\mathbf{z}}=\underset{0 \leq t \leq \frac{T}{2}-1}{\operatorname{clm}}\left(\mathbf{z}_{t}\right)$ based upon $\overline{\mathbf{y}}_{D}^{\mathrm{equ}, k}$, the $N_{S} T \times 1$ conditional mean signal vector $\underline{\overline{\mathbf{z}}}=\underset{0 \leq t \leq \frac{T}{2}-1}{\operatorname{clmn}}\left(\overline{\mathbf{z}}_{t}\right)$ where

$$
\overline{\mathbf{z}}_{t} \triangleq\left[\begin{array}{c}
\overline{\mathbf{z}}_{t}^{(1)} \\
\vdots \\
\overline{\mathbf{z}}_{t}^{\left(2 N_{S}\right)}
\end{array}\right] \in \mathbb{C}^{2 N_{S} \times 1},
$$

and the diagonal $N_{S} T \times N_{S} T$ conditional covariance matrix $\Theta_{\overline{\mathbf{z}}}^{(k)}$ delivered by the soft mapper. In the following subsections we will elaborate more on how $\underline{\widetilde{\mathbf{z}}}^{(k)}$ is produced based upon $\overline{\mathbf{y}}_{D}^{e q u, k}, \underline{\mathbf{z}}$ and $\boldsymbol{\Theta}_{\overline{\mathbf{z}}}^{(k)}$. The $\left(2 N_{S} t+i\right)$ th element in $\underline{\overline{\mathbf{z}}}$ and the $\left(2 N_{S} t+i, 2 N_{S} t+i\right)$ th element in $\Theta_{\overline{\mathbf{Z}}}^{(k)}$ are respectively evaluated by the soft-mapper for $i=1, \cdots, 2 N_{S}$, and $t=0, \cdots, T / 2-1$ as

$$
\left\{\begin{array}{l}
\overline{\mathbf{z}}_{t}^{(i)}=\sum_{\mathbf{z} \in \mathcal{X}} \mathbf{z} \mathbf{P}_{t}^{(i)}\left(\mathbf{z} \mid \mathcal{A}_{t}^{(i)}\right) \\
\theta_{i, t}^{(k)}=\sum_{\mathbf{z} \in \mathcal{X}}|\mathbf{z}|^{2} \mathbf{P}_{t}^{(i)}\left(\mathbf{z} \mid \mathcal{A}_{t}^{(i)}\right)-\left|\overline{\mathbf{z}}_{t}^{(i)}\right|^{2},
\end{array},\right.
$$

where the conditional probability function $\mathbf{P}_{t}^{(i)}\left(\mathbf{z} \mid \mathcal{A}_{t}^{(i)}\right)$ is measured as the probability that symbol $\mathbf{z} \in \mathcal{X}$ had been virtually transmitted by node $S$ at channel use $t$ from antenna $i$, given the vector $\mathcal{A}_{t}^{(i)} \in \mathbb{R}^{\log _{2}(|\mathcal{X}|) \times 1}$ of a priori LLRs about coded bits corresponding to the transmission of $\mathbf{z}_{t}^{(i)}$. We return back to the soft sub-packet combiner output, and assume that $\underline{\widetilde{\mathbf{z}}}^{(k)}$ has been produced. Then, the soft de-mapper, also benefiting from the a priori LLRs in $\mathcal{A}_{t}^{(i)}$, resorts to the $\left(2 N_{S} t+i\right)$ th component in $\widetilde{\mathbf{z}}^{(k)}$ to extract the corresponding vector of extrinsic LLRs $\mathcal{E}_{t}^{(i)} \in \mathbb{R}^{\log _{2}(|\mathcal{X}|) \times 1}$ before being re-grouped as

$$
\left[\mathcal{E}_{0}^{(1)^{\mathrm{T}}}, \cdots, \mathcal{E}_{0}^{\left(2 N_{S}\right)^{\mathrm{T}}}, \cdots, \mathcal{E}_{\frac{T}{2}-1}^{(1)^{\mathrm{T}}}, \cdots, \mathcal{E}_{\frac{T}{2}-1}^{\left(2 N_{S}\right)^{\mathrm{T}}}\right] \in \mathbb{R}^{1 \times \log _{2}(|\mathcal{X}|) N_{S} T},
$$

de-interleaved, and given to the SISO decoder. The updated extrinsic LLRs to be provided to the interleaver are formed by substracting the a-posteriori LLRs at the output of the SISO decoder from the a priori LLRs at its input. Then, the soft-mapper, depending on the constellation set and a priori information received from the interleaver, computes the conditional probabilities $\mathbf{P}_{t}^{(i)}\left(\mathbf{z} \mid \mathcal{A}_{t}^{(i)}\right)$ and fed the soft sub-packet combiner with $\underline{\overline{\mathbf{z}}}$ and $\Theta_{\overline{\mathbf{z}}}^{(k)}$. This completes one iteration, and the same processing is repeated for a preset number of iterations before hard-decision being taken by the SISO decoder. The result is a reliable estimate $\widetilde{b}$ of the transmitted packet of information bits. The estimated packet is checked, then an ACK/NACK feedback is sent back to node $S$. If it incidentally moves up to round $k+1$, the soft sub-packet combiner and softdemapper in their initial iteration will benefit from the output of the soft-mapper and interleaver in the last iteration of round $k$, respectively, as input in the current iteration.

\section{B. Soft Sub-Packet Combiner Derivation}

Pertaining to (12), the corresponding $N_{D} k T \times N_{S} T$ sub-packet ARQ transmission model is given by

$$
\underline{\mathbf{y}}^{\mathrm{equ}, k}=\underline{\mathbf{H}}^{\mathrm{equ}, k} \underline{\mathbf{z}}+\underline{\mathbf{n}}^{\mathrm{equ}, k},
$$

where

$$
\left\{\begin{array}{l}
\underline{\mathbf{z}}=\underset{0 \leq t \leq \frac{T}{2}-1}{\operatorname{clmm}}\left(\mathbf{z}_{t}\right) \\
\underline{\mathbf{y}}^{\mathrm{equ}, k}=\operatorname{clmn}_{0 \leq t \leq \frac{T}{2}-1}\left(\overline{\mathbf{y}}_{D, t}^{\mathrm{equ}, k}\right) \\
\underline{\mathbf{n}}^{\mathrm{equ}, k}=\underset{0 \leq t \leq \frac{T}{2}}{\operatorname{clm} \mathbf{n}}\left(\overline{\mathbf{n}}_{D, t}^{\mathrm{equ}, k}\right)
\end{array},\right.
$$

and $\underline{\mathbf{H}}^{\text {equ, } k}$ is a block circulant matrix whose first $N_{D} k T \times 2 N_{S}$ block column is equal to

$$
\left[\overline{\mathbf{H}}_{0}^{\mathrm{equ}, k^{\mathrm{T}}}, \cdots, \overline{\mathbf{H}}_{L_{e q u}-1}^{\mathrm{equ}, k^{\mathrm{T}}}, \mathbf{0}_{2 N_{S} \times 2 N_{D} k\left(T / 2-L_{e q u}\right)}\right]^{\mathrm{T}} .
$$

Therefore, it can be block-diagonalized in the Fourier basis as

$$
\underline{\mathbf{H}}^{\mathrm{equ}, k}=\mathbf{U}_{T / 2,2 N_{D} k}^{\mathrm{H}} \underline{\Delta}^{(k)} \mathbf{U}_{T / 2,2 N_{S} k},
$$

where $\underline{\Delta}^{(k)} \in \mathbb{C}^{N_{D} k T \times N_{S} T}$ is a block diagonal matrix holding on its diagonal the $2 N_{D} k \times 2 N_{S}$ matrices $\Delta_{0}^{(k)}, \cdots, \Delta_{T / 2-1}^{(k)}$ that can be computed for $t=0, \cdots, T / 2-1$ as

$$
\Delta_{t}^{(k)}=\sum_{l=0}^{L_{e q u}-1} \overline{\mathbf{H}}_{l}^{\mathrm{equ}, k} \exp \left\{-j \frac{4 \pi t l}{T}\right\} .
$$

As we may also be interested in expressing the memoryless single round $k$ sub-packet transmission model, we similarly to (14) get 
from (8) the following $N_{D} T \times N_{S} T$ model

$$
\underline{\mathbf{y}}^{\mathrm{equ}(k)}=\underline{\mathbf{H}}^{\mathrm{equ}(k)} \underline{\mathbf{z}}+\underline{\mathbf{n}}^{\mathrm{equ}}{ }^{(k)}
$$

where

$$
\left\{\begin{array}{l}
\underline{\mathbf{y}}^{\mathrm{equ}^{(k)}}=\underset{0 \leq t \leq \frac{T}{2}-1}{\operatorname{clm} \mathbf{n}}\left(\mathbf{y}_{D, t}^{\mathrm{equ}(k)}\right) \\
\underline{\mathbf{n}}^{\mathrm{equ}(k)}=\underset{0 \leq t \leq \frac{T}{2}}{\operatorname{clm}}\left(\mathbf{n}_{D, t}^{\mathrm{equ}^{(k)}}\right)
\end{array}\right.
$$

and $\underline{\mathbf{H}}^{\mathrm{equ}}{ }^{(k)}$ is a block circulant matrix whose first $N_{D} T \times 2 N_{S}$ block column is equal to

$$
\left[\mathbf{H}_{0}^{\mathrm{equ}^{(k)^{\mathrm{T}}}}, \cdots, \mathbf{H}_{L_{e q u}-1}^{\mathrm{equ}^{(k)^{\mathrm{T}}}}, \mathbf{0}_{2 N_{S} \times 2 N_{D}\left(T / 2-L_{e q u}\right)}\right]^{\mathrm{T}} .
$$

It can be block diagonalized in the Fourier basis to $\Upsilon^{(k)}$ as $\underline{\mathbf{H}}^{\text {equ, } k}$ in (17). By applying the DFT to both sides of (19) and (14), we respectively obtain the following single and multi-round frequency domain (FD) sub-packet ARQ transmission models

$$
\begin{aligned}
\underline{\mathbf{y}}_{f}^{\mathrm{equ}^{(k)}} & =\underline{\Upsilon}^{(k)} \underline{\mathbf{z}}_{f}+\underline{\mathbf{n}}_{f}^{\mathrm{equ}}{ }^{(k)} \\
\underline{\mathbf{y}}_{f}^{\mathrm{equ}, k} & =\underline{\Delta}^{(k)} \underline{\mathbf{z}}_{f}+\underline{\mathbf{n}}_{f}^{\mathrm{equ}, k} .
\end{aligned}
$$

By solving the optimization problem in the MMSE filtering sense related to (23), we obtain a soft estimate $\underline{\widetilde{\mathbf{z}}}_{f}^{(k)}$ about $\underline{\mathbf{z}}_{f}$ according to the following joint forward-backward filtering and signal-level sub-packet combining structure

$$
\underline{\widetilde{\mathbf{z}}}_{f}^{(k)}=\boldsymbol{\Phi}^{(k)} \underline{\mathbf{y}}_{f}^{\mathrm{equ}, k}-\boldsymbol{\Psi}^{(k)} \underline{\overline{\mathbf{z}}}_{f},
$$

where the forward filter $\boldsymbol{\Phi}^{(k)}=\operatorname{diag}\left\{\boldsymbol{\Phi}_{0}^{(k)}, \cdots, \boldsymbol{\Phi}_{T / 2-1}^{(k)}\right\}$, and backward filter $\Psi^{(k)}=\operatorname{diag}\left\{\Psi_{0}^{(k)}, \cdots, \Psi_{T / 2-1}^{(k)}\right\}$ are expressed, for $t=0, \cdots, T / 2-1$, as

$$
\left\{\begin{array}{l}
\boldsymbol{\Phi}_{t}^{k}=\frac{1}{N_{0}} \Delta_{t}^{(k)^{\mathrm{H}}}\left\{\mathbf{I}_{2 N_{D} k}+\Delta_{t}^{(k)} \mathbf{C}_{t}^{-1} \underline{\Delta}_{t}^{(k)^{\mathrm{H}}}\right\} \\
\mathbf{C}_{t}^{-1}=N_{0} \widetilde{\boldsymbol{\Theta}}_{\overline{\mathbf{Z}}}^{(k)^{-1}}+\Delta_{t}^{(k)^{\mathrm{H}}} \Delta_{t}^{(k)} \\
\Psi_{t}^{k}=\boldsymbol{\Phi}_{t}^{(k)} \underline{\Delta}_{t}^{(k)}-\frac{2}{T} \sum_{i=0}^{T / 2-1} \boldsymbol{\Phi}_{t}^{(k)} \underline{\Delta}_{t}^{(k)}
\end{array}\right.
$$

In (25), the diagonal matrix $\widetilde{\Theta}_{\underline{\overline{\mathbf{Z}}}}^{(k)}$ has been introduced as the time average of $\boldsymbol{\Theta}_{\overline{\mathbf{z}}}^{(k)}$, whose $\left(2 N_{S} t+i, 2 N_{S} t+i\right)$ th diagonal element is computed as

$$
\widetilde{\theta}_{i}^{(k)}=\frac{2}{T} \sum_{t=0}^{T / 2-1} \theta_{i, t}^{(k)}
$$

For the purpose of reducing the computational load of the proposed receiver, notice that inverting channel matrices $\left\{\mathbf{C}_{t}^{-1}\right\}$ in (25) incurs, thanks to the matrix inversion lemma [10], only a computational complexity order that is cubic against $2 N_{S}$, instead of $2 N_{D} k$ [9]. This is a critical solved issue in our analysis since the complexity order would have been increasing with the ARQ delay. Once the soft sub-packet combiner constructs the soft symbol vector $\underline{\widetilde{z}}_{f}^{(k)}$, it takes its DFT inverse to get the equalized time domain vector $\widetilde{\mathbf{z}}^{(k)}$. The rest goes with what we have already described in the previous subsection.

\section{Recursive Implementation}

To get some intuition behind the recursion process, we re-express the matrix product $\Delta_{t}^{(k)^{\mathrm{H}}} \underline{\Delta}_{t}^{(k)}$ in $(25)$ as

$$
\underline{\Delta}_{t}^{(k)^{\mathrm{H}}} \underline{\Delta}_{t}^{(k)}=\sum_{p=1}^{k} \underline{\Upsilon}^{(p)^{\mathrm{H}}} \underline{\Upsilon}^{(p)}
$$

After some manipulations, we introduce the following recursive variables $\Gamma_{t}^{(k)}=\underline{\Gamma}_{t}^{(k-1)}+\underline{\Upsilon}_{t}^{(k)^{\mathrm{H}}} \underline{\Upsilon}_{t}^{(k)}$ (with $\underline{\Gamma}_{t}^{(0)}=\mathbf{0}_{2 N_{S} \times 2 N_{S}}$ ) and $\underline{\widetilde{\mathbf{y}}}_{f}^{\text {equ }}{ }^{(\bar{k})}=\underline{\widetilde{\mathbf{y}}}_{f}^{\mathrm{equ}^{(k-1)}}+\underline{\Upsilon}^{(k)^{\mathrm{H}}} \underline{\mathbf{y}}_{f}^{\mathrm{equ}^{(k)}}$ (with $\underline{\widetilde{\mathbf{y}}}_{f}^{\mathrm{equ}^{(0)}}=\mathbf{0}_{2 N_{S} \times 1}$ ) within the equations of (25). Therefore, one may re-write (24) in a new structure as

$$
\underline{\widetilde{\mathbf{z}}}_{f}^{(k)}=\widetilde{\boldsymbol{\Phi}}^{(k)} \underline{\mathbf{y}}_{f}^{\text {equ }}{ }^{(k)}-\widetilde{\mathbf{\Psi}}^{(k)} \underline{\overline{\mathbf{z}}}_{f} .
$$

In particular, the previous backward-forward filters have now been adjusted to $\widetilde{\boldsymbol{\Phi}}^{(k)}=\operatorname{diag}\left\{\widetilde{\boldsymbol{\Phi}}_{0}^{(k)}, \cdots, \widetilde{\boldsymbol{\Phi}}_{T / 2-1}^{(k)}\right\}$ and $\widetilde{\boldsymbol{\Psi}}^{(k)}=$ $\operatorname{diag}\left\{\widetilde{\boldsymbol{\Psi}}_{0}^{(k)}, \cdots, \widetilde{\boldsymbol{\Psi}}_{T / 2-1}^{(k)}\right\}$ with

$$
\left\{\begin{array}{l}
\widetilde{\boldsymbol{\Phi}}_{t}^{(k)}=\frac{1}{N_{0}}\left\{\mathbf{I}_{2 N_{S}}-\underline{\Gamma}_{t}^{(k)} \mathbf{C}_{t}^{-1}\right\} \\
\mathbf{C}_{i}=N_{0} \widetilde{\boldsymbol{\Theta}}_{\overline{\mathbf{z}}}^{(k)^{-1}}+\underline{\Gamma}_{t}^{(k)} \\
\widetilde{\mathbf{\Psi}}_{t}^{(k)}=\widetilde{\boldsymbol{\Phi}}_{t}^{(k)} \underline{\Gamma}_{t}^{(k)}-\frac{2}{T} \sum_{t=0}^{T / 2-1} \widetilde{\boldsymbol{\Phi}}_{t}^{(k)} \underline{\Gamma}_{t}^{(k)}
\end{array} .\right.
$$

Importantly, implementing these recursions will enable us to avoid storing in memory equivalent received signals and channel frequency responses whose size gradually increase with the ARQ delay. Instead, the soft sub-packet combiner now requires to keep in memory at round $k$ (in the case of packet decoding failure) only two variables $\underline{\widetilde{\mathbf{y}}}_{f}^{\text {equ }}{ }^{(k)} \in \mathbb{C}^{2 N_{S}}$ and $\underline{\Gamma}_{t}^{(k)} \in \mathbb{C}^{2 N_{S}}$ of fixed size. Furthermore, the computational cost of the proposed recursive implementation is much more reduced compared with the direct implementation of (24), since the operations in (27) involve only small and fixed channel matrix additions and multiplications.

\section{NuMERICAL RESUlTS}

We focus on the average throughput as a meaningful performance measure influenced by the packet error rate (PER) and the system latency $\mathcal{L}$ according to

$$
\mathcal{T}_{a}=\mathcal{S} \frac{(1-\mathrm{PER})}{\mathcal{L}}
$$

where $\mathcal{L}$ is computed as the average of the ARQ system delay over a sufficiently large number of packet transmissions, and $\mathcal{S}$ is the spectral efficiency. Specifically, we assess the proposed turbo receiver scheme in terms of the average throughput via MonteCarlo simulations in comparison to turbo reception in the context of the so-called "AF cooperative relaying" (CR - AF) and "Selective DF cooperative relaying" (CR - Selective DF) protocols [3]. These protocols suggest that node $S$ broadcasts the whole packet $\mathrm{x}$ at once to both $R$ and $D$ nodes during the first TS, while only node $R$ transmits during the second TS. We infer then that in this context each packet transmission will span four time slots (or two ARQ rounds). The system parameters are shown in Table I. In all figures, the SNR corresponds to that of the direct link and is defined as

$$
\mathrm{SNR}=\frac{E_{S D} E_{b}}{N_{0}},
$$

where in general $E_{A B}=l_{A B}^{-\kappa}$ with $\kappa$ the path loss exponent and $l_{A B}$ the distance between node $A$ and node $B$. For the sake of 
Table I

Simulation Settings

\begin{tabular}{c|c} 
Parameter & Description \\
\hline \hline$T$ & 516 \\
\hline$N_{S}, N_{R}$ & 2 \\
\hline$N_{D}$ & 3 \\
\hline$L_{S R}, L_{R D}, L_{S D}$ & 3 \\
\hline Encoding & $\frac{1}{2}$-Rate Convolutional Encoder $(25,23)_{8}$ \\
\hline Interleaver size & 1040 (including tail bits) \\
\hline Modulation & QPSK \\
\hline Decoding Algorithm & Max-log-Map \\
\hline De-mapping Approximation & 3 \\
\hline Number of iterations & FD unconditional MMSE \\
\hline Filtering & 2 \\
\hline$K$ & 3 \\
\hline$\kappa$ & $0.3,0.6$ \\
\hline$d_{S R}$ &
\end{tabular}

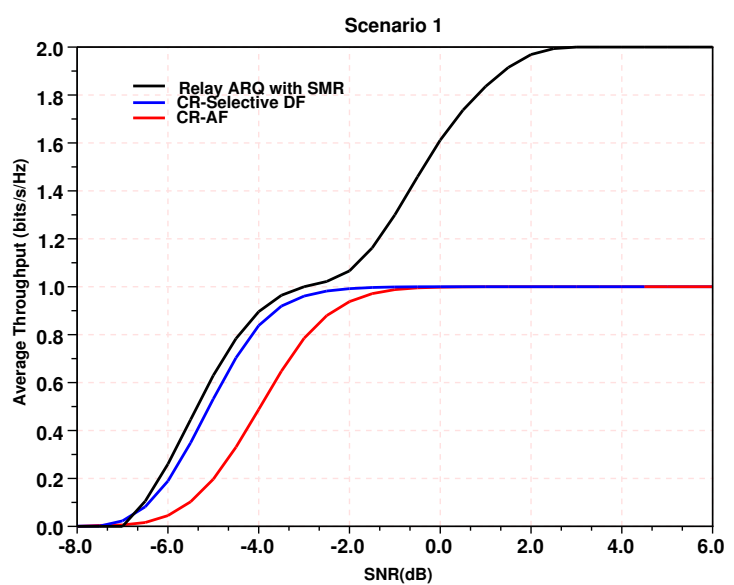

simplicity, we consider a normalized configuration in which node $S$ is located between node $R$ and node $D$ such that $l_{S D}=l_{S R}+$ $l_{R D}=1 . E_{b}$ denotes, in the absence of the relay node, the received energy at the destination node $D$ per useful bit per receive antenna.

The simulations are conducted with distinction between two scenarios. In scenario 1, the relay is closer to the source, while in scenario 2, the relay is closer to the destination. From Fig. 5 and Fig. 6, we observe that the proposed transmission strategy along with turbo sub-packet combining at the destination node lead to a better average throughput over the entire SNR region compared with turbo reception in the context of conventional ARQ-based relaying protocols. Clearly, when the latters saturate at the value of $1 \mathrm{bit} / \mathrm{s} / \mathrm{Hz}$ for SNR ratios greater than $2 \mathrm{~dB}$ due to the half-duplex constraint, the Relay ARQ with SMR scheme continues to increase in average throughput until it achieves the full rate transmission of $2 \mathrm{bit} / \mathrm{s} / \mathrm{Hz}$. Interestingly, the superiority of the proposed relaying and receiver schemes do not rely on the distance between the source and the relay node. Though CR - AF performs better than selective DF when the relay node $R$ approaches node $D$ (Fig. 6) and the opposite when node $R$ approaches node $S$ (Fig. 5), the relay ARQ with SMR attains the highest average throughput despite of the relay node position in between the two $S$ and $D$ nodes. This is justified by the fact that node $D$ succeeds in leveraging the available relaying and ARQ diversities via the proposed turbo sub-packet combining strategy in enhancing the average throughput.

\section{CONCLUSION}

In this paper, we focused on throughput-efficient relay ARQ transmissions over broadband cooperative MIMO channels. We considered a two-slot transmission strategy that, combined with signal-level sub-packet combining at the the destination side, enables to achieve attractive system average throughput compared with conventional ARQ-based cooperative relaying protocols. We also addressed the computational load of the proposed turbo receiver scheme, and provide an efficient recursive implementation that considerably reduces both the receiver's complexity and memory requirements.

\section{ACKNOWLEDGMENT}

The authors would like to thank Hatim Chergui for his valuable help, and Houda Chafnaji for some of the simulation results provided in Fig. 5 and Fig. 6.

Figure 5. Average Throughput versus SNR for $l_{S R}=0.3$.

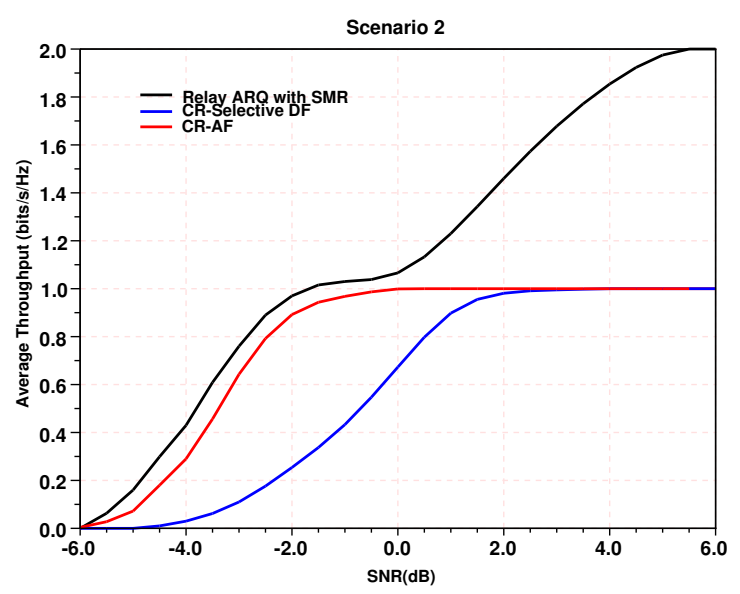

Figure 6. Average Throughput versus SNR for $l_{S R}=0.6$.

\section{REFERENCES}

[1] J. N. Laneman, G. W. Wornell, and D. N. C. Tse, "An efficient protocol for realizing cooperative diversity in wireless networks," in Proc. IEEE ISIT, Washington, DC, Jun. 2001.

[2] A. Sendonaris, E. Erkip, and B. Aazhang, "User cooperation diversity - Part I \& Part II,” IEEE Trans. Commun., vol. 51, pp. 1927-1948, Nov. 2003.

[3] H. Chafnaji, T. Ait-Idir, H. Yanikomeroglu, and S. Saoudi, "On the design of turbo packet combining schemes for relay-assisted systems over multi-antenna broadband channels," in Proc. IEEE VTC Spring, Taipei, Taiwan, May 2010.

[4] R. U. Nabar, H. Bolcskei, and F. W. Kneubuhler, "Fading relay channels: performance limits and space-time signal design," IEEE Journal Select. Areas in Commun., vol. 22, no. 6, pp. 1099-1109, Aug. 2004.

[5] Z. El-Moutaouakkil, T. Ait-Idir, H. Yanikomeroglu, and S. Saoudi, "Relay ARQ strategies for single carrier MIMO broadband amplify-and-forward cooperative transmission," in Proc. IEEE PIMRC, Istanbul, Turkey, Sep. 2010.

[6] T. Ait-Idir, and S. Saoudi, "Turbo packet combining strategies for the MIMOISI ARQ channel," IEEE Trans. Commun., vol. 57, no. 12, pp. 3782-3793, Dec. 2009.

[7] T. Ait-Idir, H. Chafnaji, and S. Saoudi, "Turbo packet combining for broadband space-time BICM hybrid-ARQ systems with co-channel interference," IEEE Trans. Wireless Commun., vol. 9, no. 5, pp. 1686-1697, May 2010.

[8] A. M. Tonello, "MIMO MAP equalization and turbo decoding in interleaved space-time coded systems,"' IEEE Trans. Commun., vol. 51, pp. 155-160, Feb. 2003.

[9] R. Visoz, A. O. Berthet, and S. Chtourou, "Frequency domain block turbo equalization for single carrier transmission over MIMO broadband wireless channel," IEEE Trans. Commun., vol. 54, no. 12, Dec. 2006.

[10] S. Haykin, Adaptive Filter Theory, 3rd Ed. Upper Saddle River, NJ: PrenticeHall, 1996. 